\title{
Compósitos minerais reforçados com madeira e borracha de pneu
}

\author{
Mineral composites reinforced \\ with wood and tire rubber
}

\author{
Douglas Lamounier Faria ${ }^{1}$, Renata Aparecida Serio Abranches ${ }^{2}$, \\ Rafael Farinassi Mendes ${ }^{3}$, Lourival Marin Mendes ${ }^{4}$, \\ Camila Soares Fonseca ${ }^{3}$, José Benedito Guimarães Júnior ${ }^{3}$
}

\footnotetext{
${ }^{1}$ Mestrando em Engenharia de Biomateriais - Departamento de Ciências Florestais - Universidade Federal de Lavras - Cx. 3037 - 37200-000 - Lavras, Minas Gerais, Brasil.

${ }^{2}$ Mestranda em Recursos Florestais - Escola Superior de Agricultura Luiz de Queiroz, ESALQ - Piracicaba, São Paulo, Brasil.

${ }^{3}$ Professor Doutor do Departamento de Engenharia - Universidade Federal de Lavras - Cx.3037 - $37200-000$ - Lavras, Minas Gerais, Brasil.

${ }^{4}$ Professor Doutor do Departamento de Engenharia Florestal - Universidade Federal de Lavras - Cx. 3037 - $37200-000$ Lavras, Minas Gerais, Brasil.

e-mail:douglas.lamounier@yahoo.com,renatabranches@yahoo.com.br, rafael.mendes@deg.ufla.br, camila.fonseca@deg.ufla.br, jose.guimaraes@deg.ufla.br, lourival@dcf.ufla.br
}

\section{RESUMO}

Nesta pesquisa, objetivou-se avaliar o potencial da madeira de Eucalyptus grandis e partículas de borracha de pneu na produção de painéis cimento-madeira. Foram utilizadas madeiras de Eucalyptus grandis com 8 anos de idade, provenientes da Companhia Mineira de Metais, localizada no município de Paracatu - MG. Para a formação dos painéis foi utilizado cimento Portland CPV-ARI/Plus de alta resistência inicial como aglutinante mineral e cloreto de cálcio $\left(\mathrm{CaCl}_{2}\right)$ como acelerador de cura do cimento. Os painéis foram produzidos com os seguintes parâmetros: dimensões de 48,0 x 48,0 x 1,5 cm, densidade nominal de $1,2 \mathrm{~g} / \mathrm{cm}^{3}$, relação madeira:cimento $(1: 2,5)$ e relação água:cimento $(1: 1,5)$. Os painéis foram prensados com pressão específica de 4 $\mathrm{MPa}$, a temperatura ambiente, tempo de prensagem/grampeamento de 24 horas e tempo de maturação de 28 dias. A análise dos resultados obtidos, em relação aos requisitos mínimos de painéis convencionais produzidos pelo processo Bison, permite afirmar que: (1) para as propriedades físicas de densidade aparente, absorção de água e inchamento em espessura todos os tratamentos atenderam as especificações; (2) para as propriedades mecânicas de ligação interna e módulo de ruptura no ensaio de flexão estática, todos os tratamentos atenderam as especificações; (3) para compressão e módulo de elasticidade no ensaio de flexão estática, nenhum dos tratamentos apresentaram valores compatíveis com o processo bison.

Palavras-chave: Painel mineral, resíduo, propriedades físico-mecânicas.

\section{ABSTRACT}

The objective of this research was to evaluate the potential of Eucalyptus grandis wood and rubber tire particles in the production of cement-wood panels. Eucalyptus grandis wood, 8 years old, was obtained from Companhia Mineira de Metais, located in the city of Paracatu - MG. For the formation of the panels, high initial strength CPV-ARI/Plus Portland cement was used as mineral binder and calcium chloride $\left(\mathrm{CaCl}_{2}\right)$ as a cement cure accelerator. The panels were produced with the following parameters: dimensions of 48,0 x 48,0 x $1,5 \mathrm{~cm}$, nominal density of $1,2 \mathrm{~g} / \mathrm{cm}^{3}$, relation wood: cement $(1: 2.5)$ and water: cement ratio (1:1.5). The panels were pressed at a specific pressure of $4 \mathrm{MPa}$, at room temperature, 24 hour pressing/stapling time, and 28 day maturation time. The analysis of the results obtained, in relation to the minimum requirements of conventional panels produced by the Bison process, allows to affirm that: (1) for the physical properties of apparent density, water absorption and swelling in thickness all the treatments met the specifications; (2) for the mechanical properties of internal bonding and modulus of rupture in the static bending test, all treatments 
met the specifications; (3) for compression and modulus of elasticity in the static bending test, none of the treatments presented values compatible with the bison process.

Keywords: Mineral panel, waste, physical-mechanical properties.

\section{INTRODUÇÃO}

De acordo com IWAKIRI [1] os painéis cimento-madeira são amplamente utilizados em construção civil nos países da Europa e Japão, em função de suas características adequadas para aplicações estruturais. No Brasil, esse material ainda não é utilizado, principalmente por questões culturais de priorizar as construções em alvenaria. Entretanto, esse cenário pode ser mudado gradativamente, por meio de políticas governamentais voltadas para construção de habitações sociais e das iniciativas dos profissionais da área de construção civil.

Painéis cimento-madeira são produtos compostos basicamente de partículas ou fibras de madeira (agregado), cimento Portland (aglomerante) e água. Aditivos químicos têm sido empregados com o propósito de reduzir o tempo de endurecimento do cimento, acelerando o desenvolvimento da resistência [2]. De acordo com Villas-Bôas et al. [3], tais painéis apresentam algumas características vantajosas como baixa permeabilidade quando comparado ao compensado, permite que sejam serrados e furados, podendo receber parafusos, além de existir grande disponibilidade de matéria-prima para sua fabricação.

O aumento da geração de resíduos sólidos é um problema que vem se agravando com o crescimento da população dos países em desenvolvimento. Dentre estes, destaca-se os resíduos pneumáticos, principalmente por terem destino final incorreto, transformou-se em sério risco ao meio ambiente. Estes materiais, em sua maioria, apresentam estrutura formada por diversos materiais como borracha, aço, nylon ou poliéster [4].

Só no Brasil são produzidos cerca de 40 milhões de pneus por ano e quase metade dessa produção é descartada nesse período. Neste sentido, a busca pelo desenvolvimento de métodos de reciclagem para este tipo de resíduo tem sido cada vez mais necessária [5].

Devido a alta disponibilidade, baixo custo, e grande quantidade dos resíduos de pneu, estes vêm se tornando uma oportunidade viável para a inserção na produção de novos produtos, ,como os compósitos minerais, uma vez que agrega valor a estes produtos por reduzir os custos de produção e o descarte no meio ambiente; proporcionando ao país benefícios tecnológicos, econômicos e ambientais. Neste sentido, sua inserção em compósitos minerais se mostra como alternativa para mitigar tal problemática.

Neste sentido, objetivo deste trabalho é avaliar as qualidades físicas e mecânicas dos painéis cimentomadeira, produzidos com partículas de Eucalyptus grandis e cimento Portland $\mathrm{CP}$, adicionando-se ainda partículas de borracha em diferentes porcentagens.

\section{MATERIAIS E MÉTODOS}

Neste estudo foi utilizado como matéria prima para produção dos compósitos minerais a madeira de Eucalyptus grandis com 8 anos de idade, provenientes da Companhia Mineira de Metais, localizada no município de Paracatu, MG. Para a formação dos painéis foi utilizado cimento Portland CP V - ARI/Plus de alta resistência inicial como aglutinante mineral e cloreto de cálcio $\left(\mathrm{CaCl}_{2}\right)$ como acelerador de cura do cimento, e ainda partículas de borracha de pneu foram acrescentadas como reforço nos compósitos minerais variando de 20 a $60 \%$ em substituição a madeira. As partículas usadas na produção dos painéis foram aquelas que passaram pela peneira de $2 \mathrm{~mm}$ e ficaram retidas na de $0,64 \mathrm{~mm}$. Foram produzidos três painéis para cada tratamento. Para a formação dos painéis, procedeu-se inicialmente o cálculo de cada um dos seguintes componentes da mistura: partículas de madeira, cimento, água e cloreto de cálcio. Na definição da quantidade para cada componente da mistura foram consideradas as seguintes proporções: relação madeira: cimento de 1:2,5; fator água : cimento de 0,25 e $6 \%$ de cloreto de cálcio em relação a massa do cimento. Os cálculos foram realizados para densidade nominal do painel de $1,25 \mathrm{~g} / \mathrm{cm}^{3}$. As dimensões do mesmo foram de $48,0 \mathrm{~cm}$ x $48,0 \mathrm{~cm} \mathrm{x}$ $1,5 \mathrm{~cm}$.

Após a pesagem de cada componente, esses foram misturados em uma betoneira para obtenção de massa homogênea. Retirada da betoneira, a massa de cada painel foi devidamente separada, pesada e distribuída aleatoriamente em placas de alumínio formadoras do colchão, untadas com óleo diesel, para facilitar a retirada do painel após sua prensagem e grampeamento. O controle da espessura dos painéis foi determinado por barras de ferro com espessuras de $1,5 \mathrm{~cm}$.

Após o carregamento dos painéis, a prensa foi fechada com pressão específica de 4,0 MPa e temperatura ambiente, realizando-se em seguida o grampeamento dos compósitos, os quais permaneceram por um 
período de 24 horas. Após esse período, os grampos foram retirados e os compósitos foram acondicionados em câmara climatizada à temperatura de $20 \pm 2^{\circ} \mathrm{C}$ e umidade relativa de $65 \pm 3 \%$ durante 28 dias, sendo então realizados os ensaios das propriedades físicas e mecânicas dos painéis.

O processo de obtenção dos corpos-de-prova foi realizado através do uso de uma serra circular. Retirou-se 4 corpos de prova por painel para absorção de água e inchamento em espessura após 24 horas de imersão [6]; 4 corpos de prova por painel para tração perpendicular [6]; 2 corpos de prova por painel, para avaliação das propriedades de flexão estática [7] e 2 corpos de prova por painel para avaliação da compressão paralela [7].

Para a análise dos dados utilizou-se o delineamento inteiramente casualizado com três repetições para cada tratamento. Foi efetuada análise de variância e regressão linear, com 5\% de significância. Para a avaliação da densidade dos painéis utilizou-se o teste de Tukey a 5\% de significância.

\section{RESULTADOS E DISCUSSÃO}

$\mathrm{Na}$ Tabela 1 estão apresentados os valores de densidade aparente para os compósitos minerais produzidos com madeira, cimento e resíduo de borracha de pneu.

Tabela 1: Densidade aparente dos painéis minerais

\begin{tabular}{cc}
\hline Tratamentos & Densidade aparente $\left(\mathrm{g} / \mathrm{cm}^{3}\right)$ \\
\hline 20 & $1,24 \mathrm{~A}(0,016)$ \\
30 & $1,25 \mathrm{~A}(0,017)$ \\
40 & $1,25 \mathrm{~A}(0,017)$ \\
50 & $1,26 \mathrm{~A}(0,005)$ \\
60 & $1,26 \mathrm{~A}(0,004)$ \\
\hline
\end{tabular}

Médias seguidas pela mesma letra, dentro da mesma coluna, não diferem estatisticamente ao nível de 5\% de significância pelo teste de Tukey.Valores entre parênteses indicam desvio padrão.

Para os valores de densidade aparente, nota-se que não houve diferenças estatísticas significativas entre os tratamentos estudados. Observa-se que todos os tratamentos ficaram acima da densidade nominal $\left(1,20 \mathrm{~g} / \mathrm{cm}^{3}\right)$, devido ao fato de que, no momento do cálculo, foram considerados $10 \%$ a mais de materiais para suprir possíveis perdas geradas durante o processo de manuseio dos componentes dos painéis no momento da homogeneização na betoneira e na divisão para a determinação da massa correspondente a cada painel antes de serem levados para a prensa. Os valores obtidos estão compatíveis com a densidade dos painéis comerciais, que segundo a BISON [8] acima de $1,10 \mathrm{~g} / \mathrm{cm}^{3}$.

Na Figura 1a observa-se o comportamento da absorção de água após 24 horas de imersão para os compósitos minerais. Nota-se efeito significativo do acréscimo de borracha de pneu nos compósito e absorção de água. $\mathrm{O}$ ponto mínimo observado pela curva descrita abaixo, ou seja, o valor de acréscimo de pneu de borracha que permitiria a menor absorção de água $(2,59 \%)$ é de aproximadamente $49 \%$.
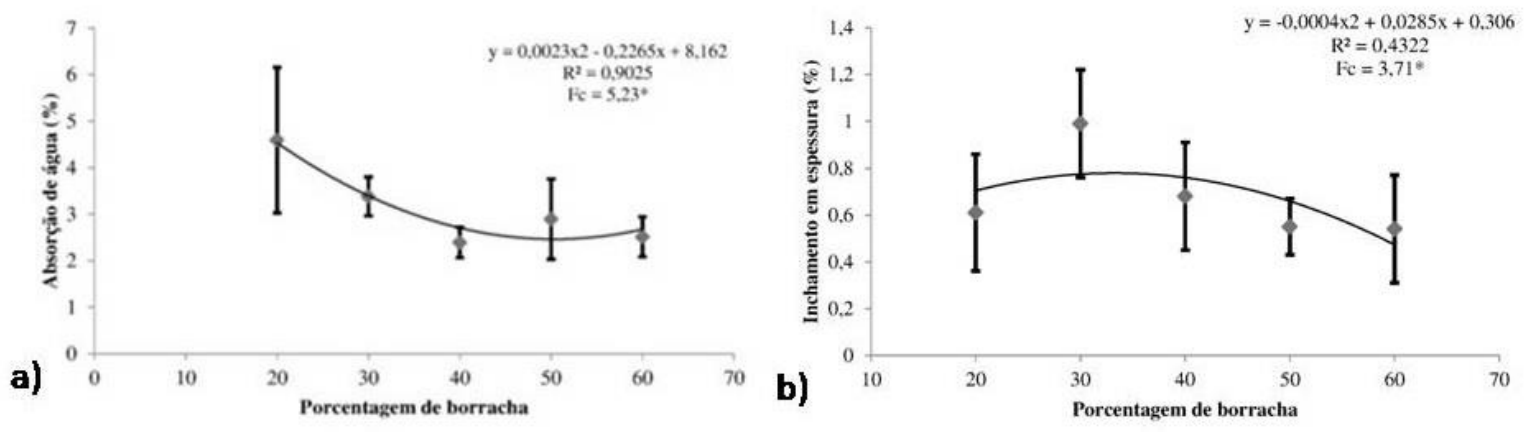

Figura 1: a) Absorção de água para os painéis minerais. b) Inchamento em espessura para os painéis minerais. $\mathrm{R}^{2}=$ Coeficiente de determinação. *Significativo $5 \%$ de significância. 
Os valores para absorção de água após 24 horas de imersão estão abaixo dos resultados obtidos por IWAKIRI [9], cujas médias variaram na faixa de 10,90\% a 14,22\%, para painéis produzidos com madeira de Eucalyptus benthamii Maiden et Cambage. OKINO [10] encontraram para painéis cimento-madeira de $\mathrm{He}$ vea brasiliensis, valores médios de absorção de água 24 horas na faixa de 15,70\% a 18,50\%. Isso pode ter ocorrido pelo fato de que o material lignocelulósico substituído tem características hidrofílicas, ao passo que o resíduo de pneu tem caráter hidrofóbico. Neste sentido, o acréscimo deste último resultaria em menor absorção de água.

Observa-se efeito significativo $(\mathrm{Fc}=3,71)$ entre inchamento em espessura após 24 horas de imersão e o acréscimo de resíduo de borracha de pneu no compósito mineral. Nota-se que o ponto de maior valor para essa propriedade é quando se aplica aproximadamente $36 \%$ de resíduo de pneu, com valor de inchamento de $0,81 \%$.

Para essa propriedade dimensional, os valores médios obtidos estão próximos da faixa de 0,71 a 0,92\% obtidos por IWAKIRI [9] para partículas de eucalipto submetidas a diferentes tratamentos químicos. De forma similar estão inferiores ao resultados observados por OKINO [10] encontraram para painéis cimento-madeira de Hevea brasiliensis, médias de inchamento em espessura 24 horas na faixa de $0,87 \%$ a $1,20 \%$. Cabe ressaltar que todos os tratamentos apresentaram-se abaixo das exigências normativa BISON [8], que estabelece valor máximo para essa propriedade de 1,8\%. A redução da propriedade de inchamento em espessura dos painéis contendo borracha de pneu pode estar associada à hidrofobicidade da borracha de pneu e ao fato de não apresentar inchamento da parede celular como acontece com a madeira, possibilitando assim melhor estabilidade dimensional.

Na Figura 2a e 2b pode-se observar o comportamento da resistência a compressão e a tração perpendicular em painéis minerais com relação ao aumento da inserção de borracha de pneu.
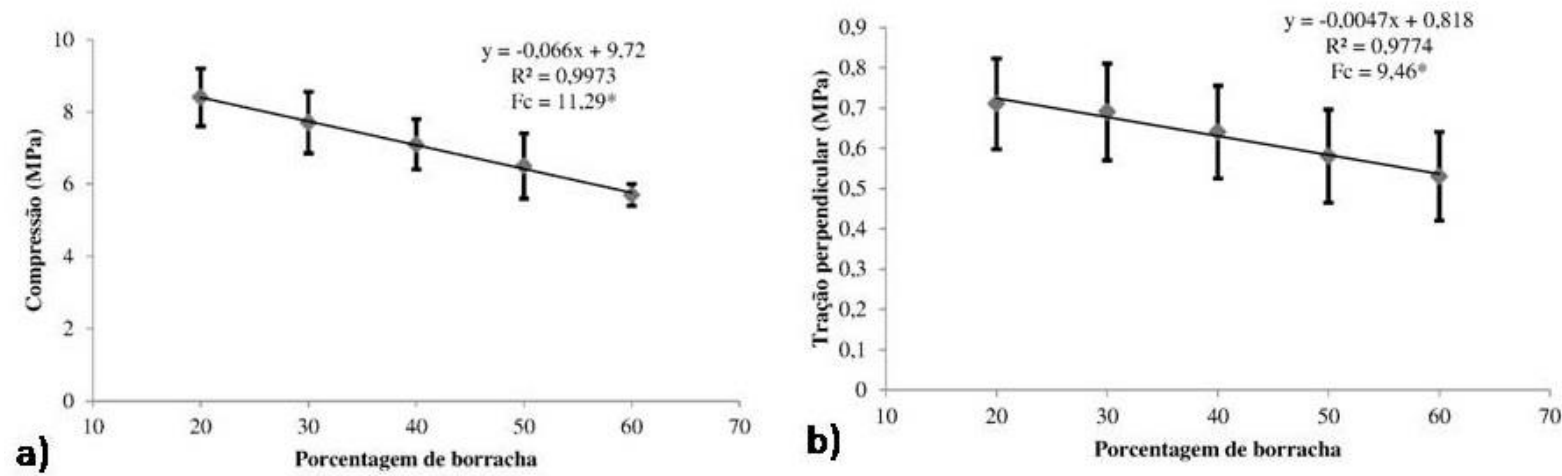

Figura 2: a) Resistência à compressão para os painéis minerais. b) Resistência a tração perpendicular para os painéis minerais. $\mathrm{R}^{2}=$ Coeficiente de determinação. *Significativo $5 \%$ de significância.

Nota-se que houve efeito significativo entre as variáveis resistência à compressão e tração perpendicular com relação ao aumento da inserção de borracha de pneu, sendo que o aumento de $1 \%$ de resíduo no compósito acarretou uma redução da resistência em compressão de 0,0066 MPa e de 0,0047 MPa para tração perpendicular. Esse comportamento pode ter ocorrido em função de a borracha adicionada ao compósito não se ligar com a madeira, nem com a matriz cimentícia. Isso poderia ter levado a aumento da porosidade do compósito e por consequência a redução de suas propriedades de resistência.

Os valores de compressão ficaram próximos ao observados por GARCEZ [11] trabalhando com compósitos cimentícios leves reforçados com partículas de madeira de Eucalyptus grandis, encontraram valores variando entre 7,34 e 14,04 MPa. Os resultados obtidos para tração perpendicular estão acima dos encontrados por MENDES [12], avaliaram painéis cimento-madeira produzidos com a madeira de Pinus oocarpa, cimento Portland CPV-ARI/Plus e densidade nominal de $1,2 \mathrm{~g} / \mathrm{cm}^{3}$ obtiveram os valores de 0,46 MPa; MATOSKI e IWAKIRI [13] trabalhando com diferentes granulometrias de partículas de Pinus spp. para a produção de painéis cimento-madeira, encontrando valores na faixa de 0,30 a 0,54 MPa. O processo BISON [8] determina valores mínimos para tração perpendicular de 0,40 $\mathrm{MPa}$. Dessa forma todos os tratamentos atendem tal normativa.

Na Figura 3a e 3b tem-se o comportamento dos compósitos reforçados com resíduos de borracha de pneu para as propriedades de Módulo de Elasticidade (MOE) e Módulo de Ruptura (MOR) na flexão estática. Para ambas as propriedades houve efeito significativo da quantidade de borracha inserida no compósito e as 
propriedades analisadas. Para MOE tem que o valor máximo para essa propriedade é quando se insere $36,60 \%$ de resíduo encontrado valor de MOE de 2074MPa. Para MOR o valor mínimo encontrado é de $10,35 \mathrm{MPa}$, utilizando a proporção de $46,74 \%$ de resíduo de pneu.
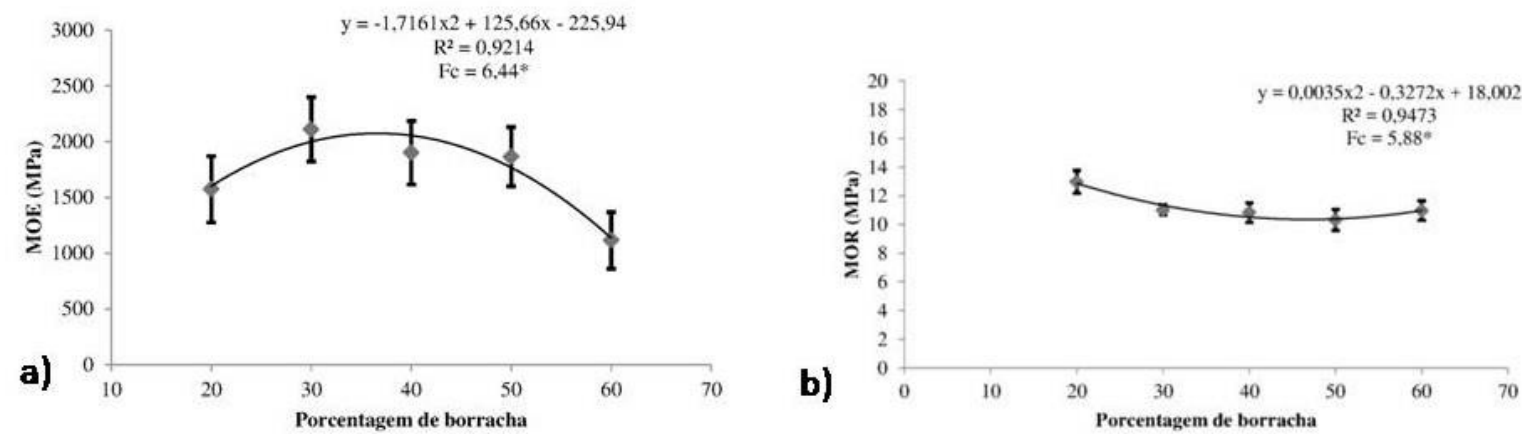

Figura 3: a) Módulo de elasticidade à flexão estática para os painéis minerais. b) Módulo de ruptura à flexão estática para os painéis minerais. $\mathrm{R}^{2}=$ Coeficiente de determinação. *Significativo $5 \%$ de significância.

Os valores encontrados para as propriedades avaliadas em flexão estática são superiores aos observados por CASTRO [14] avaliando painéis cimento-madeira de Eucalyptus saligna com diferentes aditivos químicos e métodos de formação, encontraram 1,16 a 4,02 MPa para o MOR e 816 a 2522 MPa para o MOE. O processo BISON [8] exige valores de MOE de $3000 \mathrm{MPa}$; o que não enquadra nenhum dos tratamentos nesta normativa. Já para MOR, todos os tratamento atendem as exigência da referida normativa $(9 \mathrm{MPa})$

\section{CONCLUSÕES}

As propriedades físicas dos compósitos minerais foram melhoradas com o acréscimo do resíduo de borracha, sendo que todos os tratamentos atenderam às exigências normativas. Nas propriedades mecânicas houve redução com o acréscimo de resíduo de borracha, sendo que apenas a propriedade de MOE não atendeu as exigências normativas em nenhum tratamento.

\section{AGRADECIMENTOS}

À Universidade Federal de Lavras, CAPES, CNPq e a FAPEMIG.

\section{BIBLIOGRAFIA}

[1] IWAKIRI, S., SILVA, L.S., TRIANOSKI, R., et al., "Avaliação do potencial de utilização da madeira de Schizolobium amazonicum "Paricá" e Cecropia hololeuca "Embaúba" para produção de painéis cimentomadeira”, Cerne, v.18, n. 2, pp. 303-308, abr./jun. 2012.

[2] SANTOS, R. C., MENDES, L.M., MORI, F.A., et al., "Aproveitamento de resíduos da madeira de candeia (Eremanthus erythropappus) para produção de painéis cimento-madeira", Cerne, v. 14, n. 3, pp. 241250, jul./set. 2008 .

[3] VILLAS-BÔAS, B. T., PARCHEN, C.F.A., SETSUO, I., et al., "Avaliação dos efeitos de diferentes dosagens de água/cal na resistência à compressão axial de compósitos de madeira-cimento", Matéria (R.J.), v. 22, n. 01, abril. 2017.

[4] MATTIOLI, L. M. L., et al., Plano de gerenciamento integrado de resíduos pneumáticos - PGIRPN, Fundação Estadual do Meio Ambiente: Fundação Israel Pinheiro, Belo Horizonte, MG, 44p., 2009.

[5] AYRILMIS, N., LAUFENBERG, T.L., WINANDY, J.E., et al., "Dimensional stability and creep behavior of heat-treated exterior medium density fiberboard", European Journal of Wood and Wood Products, Berlin, v. 67, n. 3, pp. 287-295, aug. 2009.

[6] AMERICAN SOCIETY FOR TESTING AND MATERIAL (ASTM). ASTM D 1037: Standart Test Methods for Evaluating Properties of Wood-Base Fiber and Particle Panel Materials.. Philadelphia (USA): Annual Book of ASTM Standards, v. 04.09 Wood, 30 p., 1998. 
[7] NORMEN FUR HOLZFASERPLATEN SPANPLATTEN SPERRHOLZ. DIN 52362: Testing of wood chipboards bending test, determination of bending strenght: Berlin, 40p, 1982.

[8] BISON WOOD-CEMENT BOARD. New York: Springer, 1978. 10p.

[9] IWAKIRI, S., TRIANOSKI, R., CUNHA, A. B., et al., "Propriedades tecnológicas de painéis cimentomadeira produzidos com partículas de eucalipto", Revista de Ciências Agroveterinárias, v. 14, n.3, pp.217223, 2015.

[10] OKINO, E. U. A., SOUZA, M.R., SANTANA, M.A.E., et al., "Chapa aglomerada de cimento-madeira de Hevea brasiliensis Müll. Arg”, Revista Árvore, v. 28, n. 3, pp. 451-457, maio/jun. 2004.

[11] GARCEZ, M. R., GARCEZ, E.O., MACHADO, A.O., et al., "Compósitos cimentícios leves reforçados com partículas de madeira de Eucalyptus grandis", Ciência da Madeira (Brazilian Journal of Wood Science), v. 8, n.2, pp. 94-100, jun. 2017.

[12] MENDES, L. M., LOSCHI, F.A.P., RAMOS, L.E., et al., "Potencial de utilização da madeira de clones de Eucalyptus urophylla na produção de painéis cimento-madeira", Cerne, v. 17, n. 1, pp. 69-75, jan./mar. 2011.

[13] MATOSKI, A., IWAKIRI, S., "Avaliação das propriedades físico-mecânicas de painéis de cimentomadeira utilizando farinha de madeira com granulometria controlada", Floresta, v. 37, n. 2, pp. 149-158, maio/ago. 2007.

[14] CASTRO, V. G., BRAZ, R.L., AZAMBUJA, R.R., et al., "Painéis cimento-madeira de Eucalyptus saligna com diferentes aditivos químicos e métodos de formação", Floresta, v. 45, n. 2, pp. 349-360, abr./jun. 2015. 